\title{
Psicoestimulantes na vida acadêmica: efeitos adversos do uso indiscriminado
}

\author{
Psychostimulants in academic life: adverse effects of indiscriminate use \\ Psicoestimulantes en la vida académica: efectos adversos del uso indiscriminado \\ Gabriel Pina PAIVA ${ }^{1}$ \\ Antonio Filipe GALHEIRA ${ }^{2}$ \\ Mateus Tomáz BORGES ${ }^{2}$ \\ ${ }^{1}$ Universidade Brasil. Faculdade de Medicina - Campus Fernandópolis 15600-000 Fernandópolis-SP, Brasil,
} Graduado pela Faculdade de Medicina da Universidade Federal do Amazonas - UFAM, Residência Médica em Neurologia no Hospital de Base da Faculdade de Medicina de São José do Rio Preto - FAMERP, Estágio em Doenças Neuromusculares no Hospital de Base da Faculdade de Medicina de São José do Rio Preto FAMERP, Título em Neurologia pela Academia Brasileira de Neurologia, Título em Neurofisiologia Clínica pela Sociedade Brasileira de Neurofisiologia Clínica ${ }^{2}$ Universidade Brasil. Faculdade de Medicina - Campus Fernandópolis 15600-000 Fernandópolis-SP, Brasil

\section{Resumo}

Introdução: Os psicoestimulantes são drogas que aumentam o estado de alerta e concentração dos seus usuários e são usados principalmente no tratamento de TDAH e narcolepsia. Entretanto, o uso dessas substâncias como potenciadores cognitivos tem crescido exponencialmente na busca por um rendimento melhor nos estudos ou no trabalho. Nesse cenário, algumas questões são levantadas a respeito dos efeitos nocivos para os usuários de psicoestimulantes. Objetivo: avaliar a prevalência do uso de psicoestimulantes e correlacionar com a utilização de outras substâncias, de modo a entender as causas e as consequências do uso indiscriminado do metilfenidato. Material e método: Revisão bibliográfica de livros, artigos e publicações sobre o tema e análise de pesquisas realizadas sobre o uso do metilfenidato para melhor compreensão do assunto. Resultados: Estudos mostraram que os principais motivos da utilização do metilfenidato são melhorar a atenção e aumentar o estado de vigília durante o consumo de álcool e de outras drogas. Conclusão: O uso indiscriminado de potenciadores cognitivos em longo prazo pode alterar vias noradrenérgicas e dopaminérgicas, predispondo transtornos obsessivos compulsivos e aditivos.

Descritores: Estimulantes do Sistema Nervoso Central; Metilfenidato; Transtorno da Personalidade Compulsiva.

\section{Abstract}

Introduction: Psychostimulants are drugs that increase the alertness and concentration of its users and are mainly used in the treatment of ADHD and narcolepsy. However, the use of these substances as cognitive enhancers has grown exponentially in the search for a better income in studies or work. In this scenario, some questions are raised about the harmful effects to users of psychostimulants. Objective: To evaluate the prevalence of psychostimulant use and to correlate with the use of other substances in order to understand the causes and consequences of the indiscriminate use of methylphenidate. Material and method: Bibliographical review of books, articles and publications on the subject and analysis of researches on the use of methylphenidate for a better understanding of the subject. Results: Studies have shown that the main reasons for the use of methylphenidate are to improve attention and increase the state of alertness during the consumption of alcohol and other drugs. Conclusion: The indiscriminate use of long-term cognitive enhancers may alter noradrenergic and dopaminergic pathways, predisposing to obsessive compulsive and addictive disorders.

Descriptors: Central Nervous System Stimulants; Methylphenidate; Compulsive Personality Disorder.

\section{Resumen}

Introducción: Los psicoestimulantes son drogas que aumentan el estado de alerta y concentración de sus usuarios y se utilizan principalmente en el tratamiento de TDAH y narcolepsia. Sin embargo, el uso de estas sustancias como potenciadores cognitivos ha crecido exponencialmente en la búsqueda de un mejor rendimiento en los estudios o en el trabajo. En este escenario, algunas cuestiones se plantean acerca de los efectos nocivos para los usuarios de psicoestimulantes. Objetivo: evaluar la prevalencia del uso de psicoestimulantes y correlacionar con la utilización de otras sustancias, para entender las causas y las consecuencias del uso indiscriminado del metilfenidato. Material y método: Revisión bibliográfica de libros, artículos y publicaciones sobre el tema y análisis de investigaciones realizadas sobre el uso del metilfenidato para una mejor comprensión del tema. Resultados: Estudios han demostrado que los principales motivos de la utilización del metilfenidato son mejorar la atención y aumentar el estado de vigilia durante el consumo de alcohol y otras drogas. Conclusión: El uso indiscriminado de potenciadores cognitivos a largo plazo puede alterar vías noradrenérgicas y dopaminérgicas, predisponiendo trastornos obsesivos compulsivos y adictivos.

Descriptores: Estimulantes del Sistema Nervioso Central; Metilfenidato; Trastorno de Personalidad Compulsiva.

\section{INTRODUÇÃO}

Os psicoestimulantes são drogas que tem por objetivo aumentar o estado de alerta e concentração e são usados para o tratamento de Transtorno do Déficit de Atenção com Hiperatividade (TDAH), narcolepsia e síndrome da fadiga crônica no idoso. Entretanto, observa-se que o uso dessas substâncias está crescendo de forma exponencial, não se limitando apenas à utilização para tratamento de patologias específicas, mas sim como potenciadores cognitivos, por pessoas sem qualquer déficit, na busca de um rendimento melhor nos estudos ou trabalho ${ }^{1}$.

As principais substâncias usadas para o aprimoramento cognitivo são: metilfenidato, lisdexanfetamina e modafinil. Dentre essas substâncias, o metilfenidato (MPH) ocupa o primeiro lugar em consumo no Brasil e no mundo ${ }^{2}$. Dados do relatório da Organização das Nações Unidas (ONU), publicado em 2008, apontam que a produção mundial deste fármaco cresceu de 2,8 toneladas em 1990 para quase 38 toneladas em $2006^{3}$.

Devido ao crescente consumo de potenciadores cognitivos, algumas questões foram levantadas a respeito da moral e da ética, bem como os efeitos nocivos para os usuários dessas substâncias, uma vez que a literatura ainda é deficiente de informações a discussão atual ${ }^{2,4}$.

Segundo Urban e $\mathrm{Gao}^{5}$, estudos de MPH em humanos e ratos evidenciaram que baixas doses desta substância melhoram as funções e cognições dependentes do córtex pré-frontal, tanto em indivíduos doentes, quanto em pessoas saudáveis. Entretanto, o MPH em doses elevadas e mal controladas causa prejuízo na atenção e no desenvolvimento de tarefas cognitivas. Com base no exposto, o objetivo desse estudo foi revisar, com base na literatura, os efeitos adversos nas vias dopaminérginas e noradrenérgicas, do uso 
indiscriminado do MPH em universitários brasileiros.

\section{MATERIAL E MÉTODO}

Trata-se de estudo descritivo e retrospectivo sobre o uso do metilfenidato (MPH) por estudantes universitários brasileiros. Foi realizada busca de artigos nos bancos de dados PubMed, LILACS, SciELO e ScienceDirect. Os descritores utilizados foram: psicoestimulantes e estudantes brasileiros/ou universitários brasileiros; metilfenidato e estudantes brasileiros /ou universitários brasileiros, nos idiomas: português, espanhol e inglês, publicados entre os anos de 2000 e 2019. Como critério de inclusão foi considerado estudos realizados com população acadêmica brasileira. Pesquisas de revisão foram excluídas. Após seleção por título e leitura dos resumos foram selecionados 12 artigos, lidos na íntegra e escolhidos para análise detalhada, por atenderam diretamente aos objetivos da pesquisa.

\section{RESULTADOS}

A Tabela 1 apresenta os artigos selecionados por autor, ano, amostra e resultados.

Tabela 1. Artigos selecionados catalogados por autor, ano, amostra e conclusões

\begin{tabular}{|c|c|c|c|}
\hline Autor & Ano & Amostra & Resultados \\
\hline Bassols et al. ${ }^{6}$ & 2008 & 168 & $\begin{array}{l}\text { 32\% dos universitários estressados } \\
\text { ( } 51,3 \% \text { da amostra) e } 15,8 \% \text { sem } \\
\text { estresse (48,7\% da amostra) } \\
\text { faziam uso de medicação }\end{array}$ \\
\hline Pereira et al. 7 & 2008 & 168 & $10,1 \%$ faziam uso de anfetaminas \\
\hline $\begin{array}{l}\text { Barros } \\
\text { Ortega }^{8}\end{array}$ & 2011 & 20 & $\begin{array}{l}\text { Tolerância às práticas que alteram } \\
\text { a neurobiologia a favor da } \\
\text { melhoria do desempenho, sem } \\
\text { ferir moralidade }\end{array}$ \\
\hline Cruz et al. ${ }^{9}$ & 2011 & 186 & $\begin{array}{l}\text { 8,6\% dos universitários relatou ter } \\
\text { feito uso de MPH em algum } \\
\text { momento }\end{array}$ \\
\hline Cesar et al. ${ }^{10}$ & 2012 & 12711 & $\begin{array}{l}\text { 0,9\% dos universitários relatou ter } \\
\text { feito uso de MPH em algum } \\
\text { momento }\end{array}$ \\
\hline Carneiro et al. ${ }^{11}$ & 2013 & 160 & $\begin{array}{l}\text { Prevalência de } 23,72 \% \text { para uso } \\
\text { indiscriminado do MPH }\end{array}$ \\
\hline Pasquini $^{12}$ & 2013 & 5128 & $\begin{array}{l}44,1 \% \text { dos universitários relatou } \\
\text { ter feito uso de MPH em algum } \\
\text { momento }\end{array}$ \\
\hline $\begin{array}{ll}\text { Mota } & \text { e } \\
\text { Pessanha }^{13} & \\
\end{array}$ & 2014 & 150 & $60 \%$ fazia do uso de metilfenidato \\
\hline Silveira et al. ${ }^{14}$ & 2014 & 156 & $\begin{array}{l}\text { 23,02\% faziam uso de MPH sem } \\
\text { prescrição }\end{array}$ \\
\hline Afonso et al. ${ }^{15}$ & 2016 & 403 & 6\% faziam uso de MPH \\
\hline Wille e Salvi ${ }^{16}$ & 2018 & 323 & 30\% relataram fazer uso do MTF \\
\hline $\begin{array}{ll}\text { Tolentino } & \text { e } \\
\text { Silva Neto } & \\
\end{array}$ & 2019 & 307 & 19,1\% faziam uso de $\mathrm{MPH}$ \\
\hline
\end{tabular}

Bassols et al. estudaram a influência do estresse no desempenho acadêmico e habilidades para tomar decisões e estabelecer uma adequada relação médico-paciente, em 168 estudantes do curso de Medicina da UFRGS. Seus resultados apontaram que $32 \%$ dos universitários estressados $(51,3 \%$ da amostra) e $15,8 \%$ sem estresse (48,7\% da amostra) faziam uso de medicação.

Os estudos de Pereira et al. ${ }^{8}$ com 168 universitários do primeiro ao último ano de Medicina da UFES apontou que 10,1\% dos universitários faziam uso de anfetaminas.

Barros e Ortega ${ }^{9}$, em estudo exploratório com 20 universitários dos cursos da área de Saúde e
Humanas observaram maior tolerância aos métodos que alteram a neurobiologia a favor do ideal social de melhoria do desempenho, não ferindo a moralidade dos entrevistados.

Um percentual de 8,6\% (16 em uma amostra de 186 universitários) foi a frequência encontrada entre alunos usuários de metilfenidato, em algum momento da vida escolar do curso de Medicina da UFBA, em pesquisa conduzida por Cruz et al. ${ }^{10}$.

Estudos conduzidos por Cesar et al. ${ }^{11}$ com 12.711 estudantes universitários de todas as áreas e em todo o país no ano de 2012 apontaram que 0,9\% dos universitários relatou ter feito uso de MPH em algum momento.

Também Carneiro et al. em estudo transversal com 160 alunos do $1^{\circ}$ ao $8^{\circ}$ período do curso de Medicina do centro Universitário de Volta Rendonda (RJ) encontraram um percentual de $23,71 \%$ para uso de MPH.

Pasquini a partir de um universo amostral que abrangeu 5128 alunos (1938 de ciências humanas, 1734 de ciências exatas e 1427 de ciências biológicas) de 30 campi universitários paulistas, sendo 18 públicos e 12 particulares, observou que $44,1 \%$ já fizeram uso de metilfenidato em algum momento na vida.

Mota e Pessanha buscaram identificar a prevalência dos universitários usuários de metilfenidato, assim como os principais motivos de utilização, formas de aquisição e possíveis efeitos colaterais. $\mathrm{O}$ estudo demonstrou uma prevalência de $60 \%$ do uso de metilfenidato nos 150 universitários dos cursos de Farmácia e Medicina de Campos dos Goytacazes, estado do Rio de Janeiro.

Silveira et al, conduziu estudos com 152 estudantes de Medicina de uma faculdade do sul do Brasil e observaram que $34,2 \%$ já haviam usado metilfenidato, sendo que $23,02 \%$ sem indicação médica. $\mathrm{O}$ número de estudantes do $6^{\circ}$ ano que fizeram uso não médico de metilfenidato foi mais de duas vezes maior do que o número de estudantes do $5^{\circ}$ ano (32,89 versus $13,15 \%$, respectivamente).

Em estudo observacional de corte transversal conduzido por Afonso et al. com 400 estudantes dos cursos de Biomedicina, Enfermagem, Farmácia e Nutrição da Faculdades Anhanguera de Brasília (DF) observou-se que $6 \%$ relatou uso de metilfenidato.

Wille e Salvi investigaram a prevalência do uso do MTF entre 323 acadêmicos de um centro universitário, em Ji-Paraná, estado de Rondônia. Segundo os autores, $30 \%$ relataram fazer uso do MTF, e destes, apenas 7,69\% apresentavam diagnóstico médico para o TDAH.

Percentual de 19,1\% para uso de MPH foi encontrado por Tolentino e Silva Neto ao identificar a frequência do uso da droga entre estudantes de $1^{\circ}$ ao $5^{\circ}$ ano do curso de Medicina de uma Faculdade de Medicina em Brasília (DF). 


\section{DISCUSSÃO}

O sistema nervoso recebe estímulos contantemente, entretanto o indivíduo é capaz de selecionar as informações mais importantes para aquele momento, ignorando as demais. Dessa forma, pode-se definir a atenção como sendo a capacidade de uma pessoa responder ao estímulo que lhe é relevante dentre todos os outros que estão presentes ${ }^{18}$.

Segundo Urban e $\mathrm{Gao}^{5}$, o córtex pré-frontal é $o$ principal centro de atenção e processamento executivo do cérebro $\mathrm{e}$ os principais neurotransmissores que atuam nessa região são a dopamina e a norepinefrina.

A função executiva desempenhada pelo córtex pré-frontal é um conjunto de processos cognitivos responsáveis por iniciar e desenvolver uma atividade com um objetivo final determinado. Para a execução correta de determinada tarefa se faz necessário manutenção de estado de alerta, atenção seletiva e sustentada ${ }^{19}$.

O Transtorno de Déficit de Atenção e Hiperatividade (TDAH) está relacionado com a desatenção, hiperatividade e impulsividade do indivíduo. $\mathrm{Na}$ fisiopatologia da doença há comprometimento das vias dopaminérgicas e noradrenérgica, com diminuição desses neurotransmissores no córtex pré-frontal. Com isso, o indivíduo apresenta uma disfunção executiva e incapacidade de manter a atenção ${ }^{20}$. Segundo Stahl $^{20}$ a sinalização excessiva por esses neurotransmissores também leva a um desequilíbrio na atenção do indivíduo por transbordamento do sinal.

$\mathrm{A}$ ativação de receptores de dopamina D1 e de receptores de noradrenalina alfa-2-a (Via mesocortico/límbica) têm importante função nos processos atencionais, de tal modo que a ação da dopamina e da noradrenalina chega a um nível ótimo quando os receptores D1 e alfa-2 são sensibilizados de forma moderada ${ }^{21}$.

Para Urban e $\mathrm{Gao}^{5}$ quando os níveis de dopamina (DA) e noradrenalina (NA) ultrapassam os níveis ideais, ocorre ativação de receptores D2, alfa-1 e beta-1 de neurônios que podem não estar relacionados com a função executiva atual, desfocando a atenção do indivíduo. Essa ativação de neurônios inespecíficos prejudica a seletividade da atenção e pode resultar em hiperatividade locomotora, distraibilidade e impulsividade.

$\mathrm{O}$ cloridrato de metilfenidato tem como mecanismo de ação a inibição de transportadores de NA (NAT) e de DA (DAT). O metilfenidato bloqueia esses transportadores de forma alostérica, interrompendo a recaptação de dopamina e noradrenalina e com isso esses neurotransmissores permanecem por mais tempo na fenda sináptica promovendo sua ação ${ }^{20}$.

$$
\text { A célula cerebral manipulada }
$$

farmacologicamente pode cursar com algumas modificações em razão de mecanismos plásticos. Estudos mostram que o lorazepam melhora a função de receptores GABA e com isso causa uma maior força sináptica e um maior potencial plástico através do aumento da atividade neuronal. Já o dextrometorfano bloqueia receptores NMDA, afetando assim o fluxo de íons e a construção e função de novos receptores no neurônio ${ }^{22}$.

Uma propriedade importante do córtex préfrontal é o seu alto nível de plasticidade. Alguns autores acreditam que isso é produto da lenta maturação desta região, outros acreditam que essa plasticidade local ocorra pela constituição protéica presente nesses neurônios.

Segundo Urban e $\mathrm{Gao}^{5}$ o uso de MPH em crianças e adolescentes que não tenham TDAH, apesar de parecerem tratadas com sucesso, incorre em déficits sutis que podem causar prejuízo em suas vidas futuras. Isso ocorre devido à inflexibilidade atencional fortalecida pelo MPH em um cérebro ainda não desenvolvido por completo, causando dificuldade do individuo em realizar mudanças de comportamento seja interpessoal ou laborativa, além de não desenvolver de forma satisfatória atividades que necessitem deslocar-se rapidamente sua atenção para outros focos. Outra anormalidade encontrada é o acometimento da sinalização glutamatérgica, aumentando a probabilidade de transtornos obsessivos compulsivos e aditivos.

O uso concomitante com outras drogas pode potencializar os efeitos a curto e longo prazo ${ }^{11}$. No entanto, a possibilidade de neuroplasticidade negativa no sistema nervoso dos usuários, pelo uso incorreto dos potenciadores, pode causar desregulação do sistema atencional, que é justamente o que se busca controlar com o uso de drogas. Consequentemente isso leva a modificação nos circuitos neuronais, predispondo o déficit de atenção ${ }^{23}$.

Além disso, a ativação de áreas de recompensa no cérebro e estimulação da sensação de prazer em pessoas com maior predisposição ao vício, leva ao comportamento de busca de estimulação constante da sensação de prazer ocasionada pelos medicamentos, por meio de doses cada vez mais elevadas da droga ou, o que é pior, abuso de drogas ilícitas que causem o mesmo efeito de prazer ${ }^{23}$.

Nesse aspecto, ressalva-se que no Brasil, o uso associado de metilfenidato ao uso de álcool e de outras drogas é regulado pela Agência Nacional de Vigilância Sanitária (ANVISA) orientando abstenção de álcool e de outras drogas em pacientes em tratamento de MPH. O uso associado ou em proximidade temporal dessas drogas pode ocasionar interferências nos mecanismos de ação do medicamento, aumentando sua toxidade e expondo os seus usuários a riscos de saúde significativos ${ }^{10}$. 


\section{CONCLUSÃO}

Com base na metodologia empregada e nos resultados encontrados conclui-se que o uso de psicoestimulantes, particularmente metilfenidato (MPH) tem sido utilizado por universitários brasileiros como estimulante cognitivo objetivando melhor desempenho escolar. O uso indiscriminado da droga pode, em longo prazo, alterar as vias dopaminérgicas e noradrenérgicas e predispor a transtornos de ansiedade, pontencializando seus efeitos colaterais se associada a outras substâncias estimulantes. O seu uso, portanto, deve ser restrito aos pacientes com indicação médica formal Transtorno de Déficit de Atenção e Hiperatividade (TDAH).

\section{REFERÊNCIAS}

1. Outram SM. The use of methylphenidate among students: the future of enhancement? J Med Ethics. 2010;36(4):198-202.

2. Freese L, Signor L, Machado C, Ferigolo M, Barros HMT. Non-medical use of methylphenidate: a review. Trends Psychiatry Psychother. 2012;34(2):110-15.

3. Ortega F, Barros D, Caliman L, Itaborahy C, Junqueira L, Ferreira CP. A Ritalina no Brasil: produções, discursos e práticas. Interface Comum Educ Saúde. 2010;14(34):499-510.

4. Itaborahy C. A Ritalina no Brasil: uma década de produção, divulgação e consumo [dissertação]. Rio de Janeiro: Instituto de Medicina Social, Universidade Estadual do Rio de Janeiro; 2009.

5. Urban KR, Gao WJ. Performance enhancement at the cost of potential brain plasticity: neural ramifications of nootropic drugs in the healthy developing brain. Front Syst Neurosci. 2014;8:38.

6. Bassols AM, Sordi AO, Eizirik CL, Seeger GM, Rodrigues GS, Reche M. Prevalência de estresse em uma amostra de estudantes do curso de medicina da Universidade Federal do Rio Grande do Sul. Rev HCPA. 2008; 28(3):153-57.

7. Pereira DS, Souza RS, Buaiz V, Siqueira MM. Uso de substâncias psicoativas entre universitários de medicina da Universidade Federal do Espírito Santo. J bras psiquiatr. 2008;57(3):188-95.

8. Barros D, Ortega F. Metilfenidato e aprimoramento cognitivo farmacológico: representações sociais de universitários. Saúde Soc. 2011;20(2):350-62.

9. Cruz TC, Barreto Junior EPDS, Gama MLM, Maia LCDM, Melo Filho MJXD, Manganotti Neto $\mathrm{O}$ et al. Uso não prescrito de metilfenidato entre estudantes de medicina da Universidade Federal da Bahia. Gazeta Méd Bahia. 2011;81(1):3-6.

10. Cesar ELR, Wagner GA, Castaldelli-Maia JM, Silveira CM, Andrade AG, Oliveira LG. Uso prescrito de cloridrato de metilfenidato $\mathrm{e}$ correlatos entre estudantes universitários brasileiros. Rev Psiq Clín. 2012;39(6):183-88.

11. Carneiro SG, Prado AST, Araújo ECJ, Moura HC, Strapasson JF, Rabelo NF et al. O uso não prescrito de metilfenidato entre acadêmicos de Medicina. Cadernos UniFOA: Edição Especial Ciênc da Saúde e Biol. 2013:53-9.

12. Pasquini NC. Uso de metilfenido (mfd) por estudantes universitários com intuitode "turbinar" o cérebro. Rev Biol Farm. 2013;9(2):107-13.

13. Mota JS, Pessanha FF, Prevalência do uso de metilfenidato por universitários de Campos dos Goytacazes, RJ. Vértices. 2014;16(1):77-86.

14. Silveira RR, Lejderman B, Ferreira PEMS, Rocha GMP. Patterns of non-medical use of methylphenidate among 5th and 6th year students in a medical school in southern Brazil. Trends Psych Psychother. 2014; 36(2):101-06.

15. Affonso RS, Lima KS, Oyama YM, Deuner MC, Garcia DR, Barboza LL et al. O uso indiscriminado do cloridrato de metilfenidato como estimulante por estudantes da área da saúde da Faculdade Anhanguera de Brasília (FAB). Infarma. 2016;28(3):166-72.

16. Wille ARF, Salvi JO. Prevalência do uso de metilfenidato em acadêmicos de um centro universitário em Ji-Paraná, Rondônia. BJSCR. 2018;24(3):13-9.

17. Tolentino JEF, Silva Neto JP. O uso off label de metilfenidato entre estudantes de medicina para aprimoramento do desempenho acadêmico. CCS 2019;30(1): Ahead of Print - AOF)

18. Lima RF. Compreendendo os mecanismos atencionais. Ciência e Cognição. 2005;6:113-22.

19. Rotta NT, Ohlweiler L, Riesgo RS. Transtorno da aprendizagem: abordagem neurobiológica e multidisciplinar. São Paulo: Artmed; 2016.

20. Stahl SM. Psicofarmacologia - bases neurocientíficas e aplicações práticas. Guanabara Koogan; 2014.

21. Brunton LL, Chabner BA, Knollmann BC. As bases farmacológicas da terapêutica de Goodman \& Gilman. 12. ed. Porto Alegre: AMGH Editora; 2002.

22. Gillick BT, Zirpel L. Neuroplasticity: an appreciation from synapse to system. Arch phys med rehabil. 2012;93(10):1846-55.

23. Gomes KM, Souza RP, Inácio CG, Valvassori SS, Réus GZ, Martins MR et al. Avaliação do ciclo claro e escuro no comportamento relacionado à ansiedade e à depressão em ratos de diferentes cidades após tratamento crônico com hidrocloridrato de metilfenidato. Rev bras psiquiatr. 2011;33(1):55-8. 
http://dx.doi.org/10.21270/archi.v8il 1.4660

\section{CONFLITO DE INTERESSES}

Os autores declaram não haver conflitos de interesse.

\section{AUTOR PARA CORRESPONDENCIA}

\section{Gabriel Pina Paiva}

gabrielneurologia@hotmail.com

Submetido em 04/04/2019

Aceito em 28/08/2019 